\title{
Signaling of NO/cGMP via IRAG
} Jens Schlossmann*

\author{
Address: Institut für Pharmakologie und Toxikologie, Universität Regensburg, D-93040 Regensburg, Germany \\ Email: Jens Schlossmann* - jens.schlossmann@chemie.uni-regensburg.de \\ * Corresponding author
}

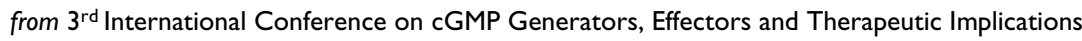

Dresden, Germany. 15-17 June 2007

Published: 25 July 2007

BMC Pharmacology 2007, 7(SuppI I):S49 doi:I0.1 I86/I47I-2210-7-SI-S49

This abstract is available from: http://www.biomedcentral.com/I47I-22/0/7/SI/S49

(c) 2007 Schlossmann; licensee BioMed Central Ltd.

Signaling by NO/cGMP/cGMP-dependent kinase I (cGKI) is important for a variety of physiological functions comprising relaxation of smooth muscle and inhibition of platelet aggregation. An important pathway of this signaling cascade includes the inositol 1,4,5-trisphosphate receptor I ( $\left.\mathrm{IP}_{3} \mathrm{RI}\right)$ associated protein cGMP kinase substrate (IRAG). This protein interacts in a trimeric macrocomplex with cGKI $\beta$ and the $\mathrm{IP}_{3} \mathrm{RI}$. To get insight into the physiological function of IRAG two different mice strains were generated by targeted deletion: (1) IRAG $G^{\Delta 12 / \Delta 12}$ with an exon 12 deletion disrupting the IRAG/IP ${ }_{3} \mathrm{RI}$ interaction. (2) IRAG-- with an exon 3 deletion generating an IRAG knockout mutant.

Analysis of IRAG ${ }^{\Delta 12 / \Delta 12}$ platelet aggregation in vitro using collagen and thrombin as agonists and Fura2 calcium measurements revealed that $\mathrm{IP}_{3} \mathrm{RI} / \mathrm{IRAG}$ interaction is essential for NO/cGMP signaling mediating inhibition of platelet aggregation. Furthermore, it was shown that $\mathrm{IP}_{3} \mathrm{RI} / \mathrm{IRAG}$ interaction is essential for the NO-dependent prevention of thrombus formation.

Relaxation of hormone-contracted aortic and longitudinal colonic smooth muscle by cGMP was abolished in IRAG $^{\Delta 12 / \Delta 12}$ mice and IRAG knockout mice indicating an essential role of IRAG for NO/cGMP-dependent smooth muscle relaxation. The vascular function of IRAG was underlined by a lack of NO-dependent blood pressure reduction in IRAG ${ }^{\Delta 12 / \Delta 12}$ mice. These studies suggest that CGKI/IRAG/IP ${ }_{3} \mathrm{RI}$ is an essential signaling pathway modulating cardiovascular functions. 